\title{
Identification of Single-Nucleotide Polymorphisms in the Mitochondrial Genome and Kelch 13 Gene of Plasmodium falciparum in Different Geographical Populations
}

\author{
Tine Kliim Nydahl, ${ }^{1,9} \dagger$ Samuel Yao Ahorhorlu, ${ }^{2} \dagger$ Magatte Ndiaye, ${ }^{3}$ Manoj Kumar Das, ${ }^{4}$ Helle Hansson, ${ }^{1,9}$ Marina Crespo Bravo, ${ }^{1,9}$ \\ Christian William Wang, ${ }^{1,9}$ John Lusingu, ${ }^{5}$ Michael Theisen, ${ }^{1,9}$ Susheel Kumar Singh, ${ }^{1,9}$ Subhash Singh, ${ }^{6}$ Susana Campino, ${ }^{7}$ \\ Ole Lund, ${ }^{8}$ Cally Roper, ${ }^{7}$ and Michael Alifrangis ${ }^{1,9 *}$ \\ ${ }^{1}$ Centre for Medical Parasitology, Department of Immunology and Microbiology, University of Copenhagen, Copenhagen, Denmark; ${ }^{2}$ Centre for \\ Tropical Clinical Pharmacology and Therapeutics, University of Ghana Medical School, University of Ghana, Ghana; ${ }^{3}$ Service de \\ Parasitologie-Mycologie, Faculté de Médecine, Université Cheikh Anta DIOP, Dakar, Senegal; ${ }^{4}$ Field Unit, National Institute of Malaria Research, \\ Ranchi, Jharkhand, India; ${ }^{5}$ National Institute for Medical Research, Tanga Centre, Tanzania; ${ }^{6}$ Indian Institute of Integrative Medicine, Jammu, India; \\ ${ }^{7}$ Faculty of Infectious and Tropical Diseases, London School of Hygiene and Tropical Medicine, London, United Kingdom; ${ }^{8}$ Genomic Epidemiology, \\ Department of Bio and Health Informatics, Technical University of Denmark, Copenhagen, Denmark; ${ }^{9}$ Department of Infectious Diseases, \\ Copenhagen University Hospital, Copenhagen, Denmark
}

\begin{abstract}
The emergence of artemisinin-resistant Plasmodium falciparum parasites in Southeast Asia threatens malaria control and elimination. The interconnectedness of parasite populations may be essential to monitor the spread of resistance. Combining a published barcoding system of geographically restricted single-nucleotide polymorphisms (SNPs), mainly mitochondria of $P$. falciparum with SNPs in the K13 artemisinin resistance marker, could elucidate the parasite population structure and provide insight regarding the spread of drug resistance. We explored the diversity of mitochondrial SNPs (bp position 611-2825) and identified K13 SNPs from malaria patients in the districts of India (Ranchi), Tanzania (Korogwe), and Senegal (Podor, Richard Toll, Kaolack, and Ndoffane). DNA was amplified using a nested PCR and Sanger-sequenced. Overall, 199 K13 sequences (India: $N=92$; Tanzania: $N=48$; Senegal: $N=59$ ) and 237 mitochondrial sequences (India: $N=93$; Tanzania: $N=48$; Senegal: $N=96$ ) were generated. SNPs were identified by comparisons with reference genomes. We detected previously reported geographically restricted mitochondrial SNPs (T2175C and G1367A) as markers for parasites originating from the Indian subcontinent and several geographically unrestricted mitochondrial SNPs. Combining haplotypes with published $P$. falciparum mitochondrial genome data suggested possible regional differences within India. All three countries had G1692A, but Tanzanian and Senegalese SNPs were well-differentiated. Some mitochondrial SNPs are reported here for the first time. Four nonsynonymous K13 SNPs were detected: K189T (India, Tanzania, Senegal); A175T (Tanzania); and A174V and R255K (Senegal). This study supports the use of mitochondrial SNPs to determine the origin of the parasite and suggests that the $P$. falciparum populations studied were susceptible to artemisinin during sampling because all K13 SNPs observed were outside the propeller domain for artemisinin resistance.
\end{abstract}

\section{INTRODUCTION}

Malaria remains a major threat to human health. Malaria has resulted in an estimated 229 million cases worldwide and an estimated 409,000 deaths in 2019; furthermore, $94 \%$ of these deaths occurred in Africa in 2019. ${ }^{1}$ The current mainstay of malaria control is the use of effective drugs as prophylaxis and treatment for malaria infections. However, throughout history, Plasmodium falciparum has shown an impressive capacity to develop resistance to nearly all of the currently available antimalarial drugs. This has significantly hindered malaria control efforts at great cost to human health. ${ }^{2}$ Therefore, a major threat in the fight against malaria is the emergence and spread of resistance to antimalarial drugs.

Historically, Southeast Asia has consecutively been the center of the emergence of resistance to antimalarial drugs and subsequent spread to sub-Saharan Africa. Conversely, resistance to chloroquine initially emerged along the Cambodia-Thailand border area in 1957 (and also in Colombia), which then spread from Thailand through Southeast Asia and India in the 1960s and 1970s. ${ }^{3}$ Chloroquine resistance was first detected in East Africa in the late 1970s; thereafter, it spread across the continent within a decade, resulting

\footnotetext{
*Address correspondence to Michael Alifrangis, Centre for Medical Parasitology, Department of Immunology and Microbiology, University of Copenhagen and Department of Infectious Diseases, Copenhagen University Hospital, Copenhagen, Denmark. E-mail: micali@sund.ku.dk

†These authors contributed equally to this work.
}

in the resurgence of malaria morbidity and mortality. ${ }^{4}$ The high levels of resistance to chloroquine forced many countries in sub-Saharan Africa to switch their first-line therapy to sulfadoxine-pyrimethamine (SP). ${ }^{5}$ However, resistance to SP developed rapidly and quickly became widespread. ${ }^{6}$ Importantly, it was later shown that SP-resistant parasites found in sub-Saharan Africa had their genetic origins in Southeast Asia. $^{7,8}$ In 2001, the WHO recommended changing to artemisinin-based combination therapies (ACTs) in countries where $P$. falciparum malaria resistance to conventional antimalarial drugs compromised in vivo efficacy ${ }^{9}$; currently, ACTs are the basis for fighting uncomplicated malaria across sub-Saharan Africa. Regretfully, P. falciparum resistance to artemisinin has been found in five countries in the Greater Mekong subregion. ${ }^{10}$ Therefore, the potential occurrence of ACT resistance spreading from Southeast Asia to subSaharan Africa, similar to what occurred with chloroquine and $\mathrm{SP}$ resistance, is imminent because three $\mathrm{K} 13$ mutations prevalent in Southeast Asia and associated with artemisinin resistance from three $P$. falciparum isolates have been discovered in China in Chinese returnees from Africa. ${ }^{11}$ Furthermore, de novo emergence of ACT resistance on the African continent is also concerning because there have been reports of imported indigenous African K13 single-nucleotide polymorphisms (SNPs) associated with delayed parasite clearance discovered in Chinese returnees from Africa. ${ }^{12}$ However, more worrying is the recent evidence of the emergence of de novo ACT resistance in parts of sub-Saharan Africa, particularly in Rwanda. ${ }^{13-18}$ 
Ariey et al. identified the propeller domain of $P$. falciparum Kelch13 (K13) as a molecular marker of artemisinin resistance by showing a strong correlation between the presence of SNPs in $\mathrm{K} 13$ and in vitro parasite survival rates and in vivo parasite clearance rates. ${ }^{19}$ Since this study, multiple groups have identified similar and additional K13 propeller SNPs, mainly in countries in Southeast Asia and, to a much lesser extent, on the African continent, enabling surveillance of emerging artemisinin resistance at the molecular level. ${ }^{20-23}$

In addition to the need for continuous research to detect the emergence of de novo ACT-resistant SNPs in sub-Saharan Africa and other malaria-endemic settings, it is crucial to understand the interconnectedness of parasite populations to trace the source of any imported infections and the geographic spread of resistance. A barcoding system identifying SNPs in mitochondria and apicoplast genomes that can distinguish the geographic origins of $P$. falciparum parasites was developed by Preston et al, ${ }^{24}$ who conducted a repetitive haplotype classification analysis using SNP variations in these two organelles combined and showed that the resulting 23-SNP barcode could predict the geographical origin of a sample with 92\% accuracy; however, it lacked representation, particularly in regions in South Asia. ${ }^{24}$ After its global validation, such a barcoding system, with data regarding molecular markers of drug resistance, could provide the means to identify and track potential increases in Asian drug-resistant $P$. falciparum parasite populations on the African continent, potentially enabling containment.

The current study examined polymorphisms in the $P$. falciparum K13 propeller in combination with SNPs in mitochondrial organelle in samples obtained from India, Tanzania, and Senegal to explore the diversity of SNPs that could distinguish these regions because limited SNP geographic barcoding data are available from these countries.

\section{MATERIALS AND METHODS}

Samples. $P$. falciparum-positive samples from Indian donors (all ages) were collected as part of a previous study performed in Dumargarhi village in the Ranchi District in the state of Jharkhand in eastern India between May 2014 and April $2016 .{ }^{25}$ Blood from donors at baseline were sampled on filter paper, and DNA was extracted using the Maxwell ${ }^{\circledR}$ 16 Blood DNA Purification Kit (Promega, Mannheim, Germany) according to the manufacturer's protocol.

Tanzanian samples were collected as part of a previous study performed in Korogwe District in Northeastern Tanzania. ${ }^{26}$ In brief, blood samples were collected during 2013 through 2016, from children admitted to Korogwe District Hospital whose blood smears were positive for $P$. falciparum. In the present study, DNA was purified from 84 of these blood samples using a QIAamp DNA Mini Kit (QIAGEN, Hilden, Germany) according to manufacturer's protocol.

Senegalese samples originated from a study conducted at 14 health facilities across Senegal. ${ }^{27}$ In brief, during the study period from July 2013 through 2014, P. falciparum-positive rapid diagnostic tests (RDTs) were collected from healthseeking individuals (all ages) and stored. Extracted DNA solutions were made available as described by Ndiaye et al. ${ }^{27}$ Samples used in the present study were from the central part (Kaolack and Ndoffane districts) and northern part (Richard Toll and Podor districts) of Senegal.
The study was approved by the Institutional Ethics Committee of the National Institute of Malaria Research, Indian Council of Medical Research, New Delhi. Ethical clearance was granted by the Medical Research Coordinating Committee and Ministry of Health and Social Welfare (Tanzania) (certificate no. NIMR/HQ/R.8a/Vol.IX/1471) with annual extension approvals. For Senegal, the National Ethics Committee for Health Research granted the RDT sampling study (ethical approval number: 000020/MSAS/DPRS/CNERS).

For all PCR tests performed during this study, positive and negative controls were included. Positive controls were DNA-purified from malaria isolates 3D7/NF54, 7G8, FCR3, and K1. Negative controls were DNA-purified from a Danish malaria-negative blood sample and ultrapure water.

Amplification of mitochondrial DNA. A 2215-bp-long sequence of the $P$. falciparum mitochondrial genome (ranging from bp positions 611 to 2825) was amplified by three sets of outer and nested PCR tests. The targeted sequence, as opposed to sequencing the whole mitochondrial genome, was chosen to save costs and after consultation with the co-authors of the Preston publication. ${ }^{24}$ Primers for amplification of mitochondrial DNA were designed using Primer-BLAST (https://www.ncbi.nlm.nih.gov/tools/primer-blast/) (Table 1) and the $P$. falciparum NF54 mitochondrial genome (NC_ 002375.1) as reference sequence. Three outer PCR tests were performed, amplifying fragments with sizes of $802 \mathrm{bp}$, $1022 \mathrm{bp}$, and $1012 \mathrm{bp}$, respectively. Three nested PCR tests amplified fragments within the outer fragments with sizes of 602 bp, 868 bp, and 898 bp, respectively. Each PCR mix with a total volume of $20 \mu \mathrm{L}$ contained $8 \mu \mathrm{L}$ of ultrapure water, $10 \mu \mathrm{L}$ of TEMPase hot start $2 \times$ Master Mix (Ampliqon, Odense, Denmark) (equivalent to a final enzymatic activity of $0.4 \mathrm{U}$ ), $1 \mu \mathrm{L}$ of forward and reverse primer mix (final concentration of $0.25 \mu \mathrm{M}$ per primer), and $1 \mu \mathrm{L}$ of sample DNA. The temperature profile used for amplification of both outer and nested PCR tests was as follows: $95^{\circ} \mathrm{C}$ for $15 \mathrm{~min}, 30$ cycles of $94^{\circ} \mathrm{C}$ for $30 \mathrm{sec}, 54^{\circ} \mathrm{C}$ for $1 \mathrm{~min}$, and $72^{\circ} \mathrm{C}$ for $2 \mathrm{~min}$, and a final extension at $72^{\circ} \mathrm{C}$ for $5 \mathrm{~min}$.

Amplification of K13 gene. The $P$. falciparum $\mathrm{K} 13$ gene was amplified by one outer PCR and two nested PCR tests according to procedures reported previously, ${ }^{28}$ with modifications resulting in a PCR product covering a 2.027-bp fragment of the K13 gene using the $P$. falciparum 3D7 kelch protein, putative partial mRNA, and complete CDS (XM_001350122.1) as reference sequence. Each $\mathrm{PCR}$ reaction mix with a total volume of $20 \mu \mathrm{L}$ contained $8 \mu \mathrm{L}$ of ultrapure water, $10 \mu \mathrm{L}$ of TEMPase hot start $2 \times$ Master Mix, $1 \mu \mathrm{L}$ of forward and reverse primer mix (final concentration of $0.25 \mu \mathrm{M}$ per primer), and $1 \mu \mathrm{L}$ of sample DNA. The temperature profile used for amplifying the outer PCR tests was as follows: $95^{\circ} \mathrm{C}$ for $15 \mathrm{~min}, 30$ cycles of $95^{\circ} \mathrm{C}$ for 30 $\mathrm{sec}, 58^{\circ} \mathrm{C}$ for $2 \mathrm{~min}$, and $72^{\circ} \mathrm{C}$ for $2 \mathrm{~min}$, and a final extension at $72^{\circ} \mathrm{C}$ for $10 \mathrm{~min}$. For the nested PCR tests, the temperature profile was $95^{\circ} \mathrm{C}$ for $15 \mathrm{~min}, 30$ cycles of $95^{\circ} \mathrm{C}$ for $30 \mathrm{sec}, 60^{\circ} \mathrm{C}$ for 1 $\mathrm{min}$, and $72^{\circ} \mathrm{C}$ for $2 \mathrm{~min}$, and a final extension at $72^{\circ} \mathrm{C}$ for $10 \mathrm{~min}$. All PCR products were visualized on $1.2 \%$ ethidium bromide agarose gels (Invitrogen, Carlsbad, CA).

Preparation for sequencing and sequence data analyses. PCR products were sequenced in both directions using the Eurofins Genomics Plate Sequencing Service (https://www. eurofinsgenomics.eu/en/custom-dna-sequencing/). Primers used for sequencing were the same primers used for amplification of nested fragments. 
TABLE 1

Primers for amplification of outer and nested fragments of the Plasmodium falciparum mitochondrial genome

\begin{tabular}{|c|c|c|c|}
\hline Primer & Sequence & Length & Position \\
\hline MitoSeq1_outer_FW & $5^{\prime}$-ACCGGTCAAAACGGAATCA-3' & $19 \mathrm{bp}$ & $510-528$ \\
\hline MitoSeq1_outer_RV & 5'-TTTCATCTTTAACTTCTGGTATGTT-3' & $25 \mathrm{bp}$ & $1287-1311$ \\
\hline MitoSeq2_outer_FW & 5'-ACTCTGTAGTITGTAGAGATGCAA-3' & $24 \mathrm{bp}$ & $1012-1035$ \\
\hline MitoSeq2_outer_RV & 5'-CATGAGGCTCGGATATAAATGA-3' & $22 \mathrm{bp}$ & 2011-2032 \\
\hline MitoSeq3_outer_FW & 5'-AAAGGAACTCGACTGGCCTA-3' & $20 \mathrm{bp}$ & 1889-1908 \\
\hline MitoSeq3_outer_RV & 5'-ACAGCTATACATCCCATAGCAAG-3' & $23 \mathrm{bp}$ & 2878-2900 \\
\hline MitoSeq1_nested_FW & 5'-TTCCCTTCTCGCCATTTGAT-3' & $20 \mathrm{bp}$ & $611-630$ \\
\hline MitoSeq1_nested_RV & 5'-ACATTTAGTTTATCACCATATCCA-3' & $25 \mathrm{bp}$ & $1208-1232$ \\
\hline MitoSeq2_nested_FW & 5'-CAGTCATACATGATGCACTAGC-3' & $22 \mathrm{bp}$ & $1120-1141$ \\
\hline MitoSeq2_nested_RV & 5'-CAGCGACAGCGGTTATACTT-3' & $20 \mathrm{bp}$ & 1968-1987 \\
\hline MitoSeq3_nested_FW & 5'-TTAACGCCTGACATGGATGG-3' & $20 \mathrm{bp}$ & 1929-1948 \\
\hline MitoSeq3_nested_RV & 5'-TGACTAATTACTCCAAAAGCAGGT-3' & $24 \mathrm{bp}$ & 2802-2825 \\
\hline
\end{tabular}

Sequencing data obtained from Eurofins Genomics were analyzed using sequencing data analysis software (CLC Main Workbench version 7.8.1; QIAGEN Bioinformatics, Aarhus, Denmark). Mitochondrial sequences were assembled to the NCBI reference sequence NC_002375.1 ( $P$. falciparum mitochondrion, complete genome). K13 sequences were assembled to the $\mathrm{NCBI}$ reference sequence XM_001350122.1 ( $P$. falciparum 3D7 Kelch protein, putative partial mRNA, complete CDS). All mtDNA sequences to perform multiple sequence alignments were retrieved from the NCBI database. The alignments were performed using MEGA (https://www.megasoftware.net/).

\section{RESULTS}

Using a subset of $P$. falciparum-positive samples from India $(N=168)$, Tanzania $(N=84)$, and Senegal $(N=176)$, a 2215bp-long sequence of the $P$. falciparum mitochondrial genome as well as the $\mathrm{K} 13$ gene (covering $2.027 \mathrm{bp}$ ) were sequenced. For the mitochondrial genome, 236 sequences were successfully generated: 93 from Ranchi, India; 48 from Korogwe, Tanzania; and 24 from Podor District, 24 from Richard Toll, 24 from Kaolack, and 24 from Ndoffane in Senegal. For the K13 gene, 199 PCR-positive samples were successfully sequenced and assembled from the different sites: 92 from Ranchi, India; 48 from Korogwe, Tanzania; and 6 from Podor District, 5 from Richard Toll, 24 from Kaolack, and 24 from Ndoffane in Senegal.

SNP findings in the mitochondrial genome in $P$. falciparum-positive samples from India, Tanzania, and Senegal. Regarding the Indian samples, for 19 of 93 (20.4\%) samples, no SNPs in the fragment of the mitochondrial genome were observed. For the remaining samples, five different SNPs were detected (Table 2): A827G, G1367A, G1511A, G1692A, and T2175C. The most prevalent SNPs were T2175C and G1367A, with prevalence rates of $32.3 \%$ (30/93) and 33.3\% (31/93), respectively. Additionally, the SNPs A827G and G1511A were jointly detected at a low prevalence $(N=4)$. Finally, the G1692A SNP, with a detection rate of $18.8 \%$, was as prevalent in the Tanzanian and Senegalese samples.

Regarding the Tanzanian samples, for $62.5 \%$ (30/48) of the samples, no SNPs were found in the studied mitochondrial genome fragment; however, 12 different SNPs were detected in the remaining $18(37.5 \%)$ samples (Table 2). The most prevalent SNP was the single SNP G1692A, which was detected in
$10.4 \%(5 / 48)$ of the samples, including one sample with an additional G2658A SNP. Additionally, G863T was detected in $4.2 \%(2 / 48)$ of the samples, including one sample with additional SNPs in T2119A and G2383A.

Regarding the Senegalese samples, of a total of 96 samples successfully sequenced, 60 (62.5\%) were not expressing any

TABLE 2

Identified SNPs and SNP combinations in the Plasmodium falciparum mitochondria genome (fragment bp position 611-2825) in samples collected from India, Tanzania, and Senegal

\begin{tabular}{|c|c|c|c|c|c|c|}
\hline \multirow{2}{*}{$\begin{array}{l}\text { Country } \\
\text { Study site }\end{array}$} & \multirow{2}{*}{$\frac{\text { India }}{\text { Ranchi }}$} & \multirow{2}{*}{$\frac{\text { Tanzania }}{\text { Korogwe }}$} & \multicolumn{4}{|c|}{ Senegal } \\
\hline & & & Podor & Richard Toll & Kaolack & Ndoffane \\
\hline $\begin{array}{l}\text { Sample size } \\
\text { SNP positions }\end{array}$ & 93 & 48 & 24 & 24 & 24 & 24 \\
\hline G1692A & 9 & 5 & 10 & 4 & 6 & 8 \\
\hline G1367A & 31 & & & & & \\
\hline T2175C & 30 & & & & & \\
\hline C1637A & & & & & 4 & 3 \\
\hline A827G & 4 & & & & & \\
\hline G1511A & 4 & & & & & \\
\hline G1692T & & & & 2 & & 2 \\
\hline G863T & & 2 & & & & \\
\hline А939T & & & 1 & & & 1 \\
\hline T1214C & & 2 & & & & \\
\hline G770A & & 1 & & & & \\
\hline C772T & & 1 & & & & \\
\hline A848G & & & 1 & & & \\
\hline G1076A & & & & 1 & & 1 \\
\hline T1100C & & 1 & & & & \\
\hline C1108T & & & 1 & & & \\
\hline G1397A & & & & & & 1 \\
\hline T1426C & & 1 & & & & \\
\hline T1887C & & & & & & 1 \\
\hline T2034A & & 1 & & & & \\
\hline T2119A & & 1 & & & & \\
\hline T2124C & & & & & & 1 \\
\hline G2383A & & 1 & & & & \\
\hline G2407A & & 1 & & & & \\
\hline G2535A & & & & & 1 & \\
\hline G2658A & & 1 & & & & \\
\hline SNP combinations & & & & & & \\
\hline C1637A, G1692A & & & & & 4 & 3 \\
\hline A827G, G1511A & 4 & & & & & \\
\hline A939T, G1692A & & & 1 & & & 1 \\
\hline G1076A, G1692A & & & & & & 1 \\
\hline G1692A, T1887C & & & & & & 1 \\
\hline G1692A, G2535A & & & & & 1 & \\
\hline G1692A, C1108T & & & 1 & & & \\
\hline G1692A, G2658A & & 1 & & & & \\
\hline G863T, T2119A, G238 & $383 A$ & 1 & & & & \\
\hline
\end{tabular}


SNPs in the sequenced mitochondrial fragments. In the remaining samples, 11 different SNPs were detected (Table 2). The G1692A SNP was detected in $29.2 \%(28 / 96)$ of the samples, and $\mathrm{G} 1692 \mathrm{~T}$ was detected at a low prevalence $(N=4 ; 4.2 \%)$. The second-most prevalent SNP in the Senegalese samples, C1637A, was detected in 7.3\% (7/96) of the samples. Large variations in the complexity of SNPs in the limited number of samples were observed among the four sites, whereas the northern districts of Podor and Richard Toll had four and three different SNPs observed, respectively (and only sharing G1692A), and the central districts of Ndoffane and Kaolack districts had eight and three different SNPs, respectively (sharing G1692A and C1637A). The combination of SNPs varied between the sites; C1637A/G1692A was observed in the Kaolack $(N=4)$ and Ndoffane $(N=3)$ districts, whereas the A939T/G1692A combination was observed in the Podor and Ndoffane districts $(N=1$ for both sites). Except for the G1692A, which was prevalent in all countries, there was complete differentiation of the SNPs identified in Tanzania and Senegal.

Diversity of constructed haplotypes in the mitochondrial genome in $\boldsymbol{P}$. falciparum-positive samples from India, including published data. Haplotypes of the $P$. falciparum mitochondria genome originating from eight locations covering various malaria endemic areas in India, including Jharkhand, were studied by Tyagi et al. ${ }^{29-31}$ Because the present study only explored a fragment of the mitochondrial genome (2215-bp fragment ranging from bp position 611 to 2825), the data are not completely comparable. However, focusing on this fragment only, a map of diversity of constructed haplotypes across states in India can be drawn (Figure 1 and Table 2). The current study identified five different haplotypes in samples originating from Jharkhand (shown as haplotypes 1 [as the reference)], 3, 4, 5, and 6 in Figure 1). Except for haplotype 6 (A827G/G1511A), all these haplotypes were identified in the limited sample set $(N=8)$ from Jharkhand. ${ }^{29-31}$ Furthermore, some regional differences were observed; for instance, in the eastern states of Jharkhand, Odisha, Assam, and Chhattisgarh, haplotypes 4 and 5 were exclusively found, but haplotypes 1 and 3 seemed to be distributed across most of the study sites in India.

SNPs in the $P$. falciparum K13 gene in samples from India, Tanzania, and Senegal. In samples from India, one SNP (A566C, resulting in a K189T change) in the K13 gene was detected in $17.4 \%$ (16/92) of samples (Table 3). Similarly, in the Tanzania samples, the A566C was prevalent in $10.4 \%$ (5/48), whereas an additional K13 SNP at G523A (resulting in an A175T change) was detected in one sample. Regarding the Senegalese samples, in addition to detecting the A566C SNP in a total of $22.0 \%(13 / 59)$ of the samples, other single SNPs at C521T (A174V) and G764A (R255K) were detected in two separate samples.

\section{DISCUSSION}

The interconnectedness of malaria parasite populations is important to determine when attempting to trace the source of imported infections and exploring the geographic spread of antimalarial drug resistance. The present study used a

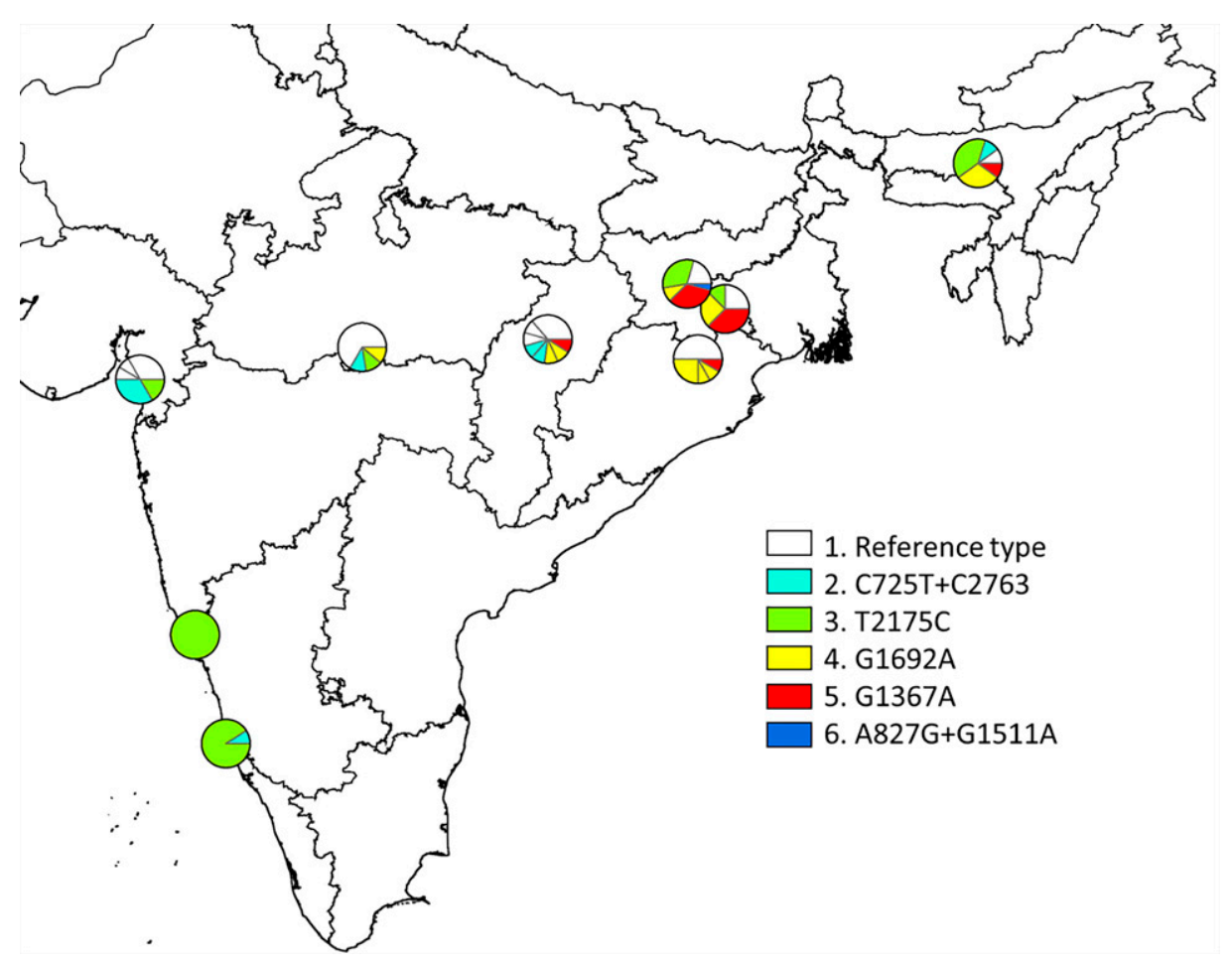

FIGURE 1. Diversity of constructed haplotypes in the mitochondrial genome (2215-bp fragment ranging from bp position 611 to 2825) in Plasmodium falciparum-positive samples from India and published data. ${ }^{20}$ The polymorphic single-nucleotide polymorphisms (SNPs) (i.e., those seen in more than two samples in the total sample set) were as follows: C725T, A827G, G1367A, G1511A, G1692, T2175C, and C2763T. These were seen in six major haplotypes:1, reference haplotype; 2, C725T + C2763T; 3, T2175C; 4, G1692A; 5, G1367A; and 6, A827G+G1511A. Minor SNPs (underlined) were grouped with the major haplotypes they otherwise resembled as follows: 1a, Ref $+\mathrm{C} 2298 \mathrm{~T}(N=1) ; 1 \mathrm{~b}, \operatorname{Ref}+\mathrm{A} 1790 \mathrm{G}(N=1) ; 1 \mathrm{c}, \operatorname{Ref}+\mathrm{A} 1441 \mathrm{~T}(N=$ 1); 1d, Ref + A959G $(N=1) ; 2 a, C 725 T+C 2763 T+C 725 T(N=2) ; 3$, no minor variants; 4a, G1692A + G728A $(N=2) ; 4 b, G 1692 A+C 1637 A(N=1)$; 4c, G1692A + C1141T $(N=1) ; 5 a, G 1367 A+G 936 T(N=1)$; and 6, no minor variants. This figure appears in color at www.ajtmh.org. 
TABLE 3

Identified SNPs in the P. falciparum $\mathrm{k} 13$ gene in samples collected from India, Tanzania, and Senegal

\begin{tabular}{|c|c|c|c|c|c|c|c|}
\hline Country & & India & Tanzania & & & negal & \\
\hline Study site & & Ranchi & Korogwe & $\begin{array}{l}\text { Podor } \\
\text { District }\end{array}$ & $\begin{array}{c}\text { Richard } \\
\text { Toll }\end{array}$ & Kaolack & Ndoffane \\
\hline Sample size & SNP & 92 & 48 & 6 & 5 & 24 & 24 \\
\hline K189T & A566C & 16 & 5 & 1 & 3 & 3 & 6 \\
\hline A175T & G523A & & 1 & & & & \\
\hline A174V & C521T & & & & & & 1 \\
\hline R255K & G764A & & & & 1 & & \\
\hline
\end{tabular}

barcoding system to identify SNPs in mitochondria genomes; this has been suggested as a way to distinguish the geographic origins of $P$. falciparum parasites. $^{24}$

Regarding the sample from India, limited diversity was observed, and the most prevalent SNPs were G1367A and T2175C. The G1367A SNP was absent in our samples from Senegal and Tanzania; to the best of our knowledge, they have not been detected in sufficient numbers in samples other than those from India and Bangladesh. ${ }^{29,32}$ The T2175C SNP is prevalent in many Asian settings. ${ }^{32}$ Therefore, these SNPs may be geographically informative. Additionally, the present study identified two other SNPs in combination that were distinctive in the Indian samples (A827G and G1511A). To the best of our knowledge and based on the results of another study, ${ }^{32}$ these two SNPs are novel and may be geographically restricted. G1692A was detected in 9.7\% (9/93) of the Indian samples. This is a quad-allelic locus that has been detected in many geographical areas, including Brazil, Nigeria, and Cameroon. ${ }^{33}$ During the present study, it was also prevalent in Tanzania and Senegal.

SNPs/haplotypes of the $P$. falciparum mitochondria genome originating from eight locations covering various malaria-endemic areas in India, including Jharkhand, were studied by Tyagi et al. ${ }^{29-31}$ By combing these data with constructed haplotype data from the present study, some interesting regional differences were observed; in particular, haplotypes 4 and 5 were only prevalent in the eastern states of India. This may imply that even regional haplotype differences within a country like India can be recognized. However, further studies are necessary to confirm this observation.

The generally higher genetic diversity in the African isolates compared with the Indian isolates is consistent with suggestions that $P$. falciparum has already infected humans and expanded greatly before they migrated out of Africa and followed our ancestors during their expansion throughout the tropics. ${ }^{34-37}$ Studies suggest that this human migration out of Africa produced sequential genetic bottlenecks for $P$. falciparum, explaining the lower genetic diversity outside of Africa, ${ }^{35,38}$ where Tanabe et al. showed a strong negative correlation between the population genetic diversity of $P$. falciparum and geographic distance from sub-Saharan Africa. ${ }^{35}$

Because of this higher genetic diversity of $P$. falciparum in Africa, it will most likely be more difficult to identify geographically restricted SNPs based on mitochondria in African countries, as suggested by the findings of this study. Preston et al. also experienced difficulty discriminating $P$. falciparum samples within the African continent. ${ }^{24}$ Moreover, the different numbers of mitochondrial SNPs observed between the various sites in Senegal may further indicate the genetically diverse nature of the parasite in an African setting. Therefore, to increase the possibility of discriminating the geographic origin of samples from different parts of Africa, a denser data representation of mitochondrial polymorphisms from this continent is needed. In addition, it is important to explore the diversity of apicoplast genomes, as previously suggested. ${ }^{39}$ Alternatively, a fully comprehensive representation and possible geographic discrimination of $P$. falciparum populations across the African continent may be achieved only by full genome-wide studies, as recently shown by AmambuaNgwa et al. ${ }^{40}$ However, the information gained as compared with that of the limited sequencing of mitochondria (and apicoplast) genomes remains to be elucidated.

The present study did not identify any SNPs in K13 associated with artemisinin resistance, which is largely in line with some studies that described polymorphisms in $P$. falciparum in samples from these same countries. A lack of SNPs in K13 has been observed during studies performed in the northeastern parts of India (Tripura, Mizoram, Arunachal Pradesh, ${ }^{41}$ and Odisha ${ }^{42}$ ). However, compared to other further northern regions of India (e.g., West Bengal and Arunanchal Pradesh), low artemisinin resistance validated K13 mutations (F446I, R539T, and $\mathrm{R} 561 \mathrm{H}$ ), and some nonvalidated candidates (e.g., A481V, N672S, and A675V) have been observed. ${ }^{43} \mathrm{Sim}-$ ilar to the present study, the lack of SNPs in K13 associated with artemisinin resistance has been observed by studies performed in western Senegal (Dakar) $22,44,45$ and eastern Tanzania (Dar es Salaam). ${ }^{22}$ The SNPs detected in the aforementioned studies were similar to the SNPs detected during the present study, and all were different from the SNPs associated with artemisinin resistance observed in Southeast Asia. The most prevalent K13 SNP found in our study was A566C (resulting in a K189T change), which was identified in samples from India (17.4\%), Tanzania (10.4\%), and Senegal (22.0\%); however, the other detected SNPs occurred only in one sample each. The A566C SNP is geographically widespread and has been detected in Uganda, ${ }^{46}$ Senegal, ${ }^{44}$ Malawi, ${ }^{39}$ the China-Myanmar border, ${ }^{47}$ Bangladesh, ${ }^{48}$ and Northeastern India. ${ }^{41}$ The $P$. falciparum $\mathrm{K} 13$ gene consists of three domains: a Plasmodium-specific domain, a BTB-POZ domain, and a Kelch propeller domain. ${ }^{19}$ The A566C SNP is positioned in the N-terminal Plasmodium-specific domain; however, it is unknown why this mutation is so widespread.

Although there is, to our best knowledge, no conclusive evidence of the spread of artemisinin-resistant $P$. falciparum phenotypes of Southeast Asian origin to sub-Saharan Africa, the potential risk of the spread of the slow parasite clearance phenotype in Southeast Asia to Africa has been corroborated by reports of three mutations in the Pfk13 propeller domain associated with artemisinin resistance (M476I, R539T, P553L) in three isolates from $P$. falciparum-infected cases discovered in individuals who returned from Africa to China between 2012 and $2015 .^{11}$

There have been some recent reports of the emergence and increased prevalence in East Africa of some indigenous SNPs in the K13 propeller domain associated with delayed parasite clearance in Southeast Asia. One such study was performed in Kibiti District in Eastern Tanzania in 2019; it identified several de novo mutations in the $\mathrm{K} 13$ gene, but at a low frequency (1/422; $0.24 \%$ ), including the $\mathrm{R} 561 \mathrm{H}$, a mutation that has been validated for delayed parasite clearance in Southeast 
Asia. ${ }^{14}$ Another study by Bergman et al. recently reported five separate nonsynonymous polymorphisms in eight isolates from southern Rwanda; three of those carried the $\mathrm{R} 561 \mathrm{H}$ SNP and 2 harbored the candidate polymorphisms C469F and $\mathrm{A} 675 \mathrm{~V} .^{13}$ They observed a significant increase in the number of isolates bearing nonsynonymous K13 SNPs over the past 10 years. Furthermore, a study performed in Masaka in central Rwanda in 2019 reported 19 of 257 (7.4\%) patients with the Pfk13 R561H SNP, and phylogenetic analysis suggested it has arisen de novo with ongoing lineage expansion. This could mark the onset of K13-based resistance to artemisinin in that country, and it could have potential implications for the efficacy of ACTs in Africa. ${ }^{16}$ Most recently, Uwimana et al. reported the presence of Pfk13 R561H SNPs in 28 of $218(13 \%)$ and P571L in 2 of $218(1 \%)$ pretreatment samples of Rwandan children with $P$. falciparum monoinfection and fever treated with artemether-lumefantrine. They observed that 8 of 51 (16\%) participants in Masaka and 12 of 82 (15\%) participants in Rukara had positive parasitemia according to microscopy on day 3 , and this was strongly correlated with pretreatment harboring of de novo Pfk13 R561H SNPs in Masaka. They confirmed evidence of emerging partial artemisinin resistance in Rwanda even though PCR-corrected efficacies for artemether-lumefantrine at their three study sites were 97.0\% (95\% Cl, 88-100\%) in Masaka, 93.8\% (95\% Cl, $85-98 \%)$ in Rukara, and $97.2 \%(95 \% \mathrm{Cl}, 91-100 \%)$ in Bugarama. $^{17}$

Additionally, Tacoli et al. reported two candidate K13 resistance mutations (P574L and A675V) in southern Rwanda that are prevalent in Southeast Asia and linked with delayed parasite clearance; they suggested that demonstrating correlations with local response to therapy and in vitro resistance assays is required for further monitoring of $\mathrm{K} 13$ polymorphisms in their study setting. ${ }^{15}$ Furthermore, Asua et al. recently reported an increased spread of $P$. falciparum $\mathrm{K} 13$ SNPs $469 Y$ (23\%) and 675V (40\%), which are associated with artemisinin-delayed parasite clearance, at multiple sites in northern Uganda. ${ }^{18}$ Finally, Lu et al. reported artemisininresistant $P$. falciparum isolates indigenous to Africa with the K13 mutation M579I in a Chinese returnee from Equatorial Guinea. ${ }^{12}$

It is important to realize that SNPs that are associated with resistance in Southeast Asia may not be the sole determinants of resistance in Africa or other areas of the world. The independent emergence of artemisinin resistance in Myanmar ${ }^{48}$ and East Africa ${ }^{13-18}$ highlights the possibility that artemisinin resistance could also emerge independently in other parts of Africa. Such de novo emergence of resistance will possibly be caused by similar or different mutations in the K13 gene or other genes conferring resistance similar to that in Southeast Asia. However, it may take a long time to register any widespread decline in the clinical response to ACTs in Africa as a result of de novo or imported K13 SNPs validated or associated with delay parasite clearance because of the high degree of immunity resulting from repeated exposure to malaria parasites that rarely manifest as disease symptoms in older age groups. ${ }^{49}$ Therefore, parasites with resistant phenotypes can potentially be masked in these high-transmission areas. Moreover, in high-transmission areas, polyclonal infections are often encountered and parasites with different phenotypes (such as drug-resistant and drug-sensitive) often recombine to increase parasite diversity, ${ }^{50}$ resulting in a dilution of drug-resistant phenotypes. Combined, these conditions would most likely cause delays in confirmatory reports describing associations between mutant genotypes and in vivo artemisinin resistance in Africa.

In addition to polymorphisms in $\mathrm{K} 13$, mutations in other genes may be involved in conferring resistance to artemisinin in $P$. falciparum. A recent study found increased ring-stage survival rates for $P$. falciparum isolates from Cambodia that harbored no K13 mutations. ${ }^{51}$ Another study performed in the United Kingdom reported K13-independent treatment failure for imported cases of $P$. falciparum malaria that had been treated with artemether-lumefantrine. ${ }^{52}$ Adams et al. reported the association of $P$. falciparum adaptor protein complex $2 \mathrm{mu}$ subunit (Pfap2mu) and $P$. falciparum ubiquitin-specific protease 1 (Pfubp1) genotypes with ACT-delayed parasite clearance in Ghana. ${ }^{53}$ Amambua-Ngwa et al. considered that strong identity by descent in Pfap2mu in Ghana and Malawi requires increased vigilance against $A C T$ efficacy..$^{40}$ Ofori et al. reported reduced in vitro sensitivities to amodiaquine, dihydroartemisinin, and mefloquine in Ghana. ${ }^{54}$

The findings of these studies indicate that artemisinin resistance monitoring should not focus entirely on K13 polymorphisms, and that efforts should be made to identify genetic loci involved in conferring artemisinin resistance in the absence of $\mathrm{K} 13$ mutations. Because it remains plausible that the emergence of artemisinin resistance in Africa could be multifaceted, it is important to combine molecular monitoring of resistance with routine assessment of the in vivo and in vitro efficacy of ACTs.

\section{CONCLUSIONS}

None of the SNPs detected in the K13 gene from samples collected in India, Tanzania, and Senegal are likely to be associated with artemisinin resistance. Some of the SNPs (T2175C and G1367A) detected in the mitochondrial genome of samples from Ranchi, India, seem geographically restricted to South Asia. Furthermore, a number of SNPs detected are reported here for the first time and may be geographically restricted. In summary, the findings of this study are of relevance to artemisinin resistance containment activities because the data suggest that the $P$. falciparum populations in the studied areas were highly susceptible to artemisinin at the time of sampling. Moreover, this study somewhat supports the use of mitochondria SNPs as part of a geographic barcode to determine the origin of infections. The results also suggest that including diversity of the apicoplast genome is most likely necessary.

Received March 18, 2021. Accepted for publication May 30, 2021.

Published online July 16, 2021.

Acknowledgments: We are grateful to all the participants who participated in the various studies in India, Tanzania, and Senegal.

Financial support: This work was supported by Building Stronger Universities Phase 3 (BSU3) programme (at University of Ghana and Kilimanjaro Christian Medical College) and by the DANIDA Fellowship Centre at the Danish Foreign Ministry. The work was also financially supported by the Wedell-Wedellsborgs Fond (Denmark) and the Aase og Ejnar Danielsens Fond (Denmark).

Authors' addresses: Tine Kliim Nydahl, Helle Hansson, Marina Crespo Bravo, Christian William Wang, Michael Theisen, Susheel Kumar Singh, and Michael Alifrangis, Centre for Medical Parasitology, 
Department of Immunology and Microbiology, University of Copenhagen and Department of Infectious Diseases, Copenhagen University Hospital, Denmark, E-mails: tinenydahl@gmail.com, hellehan@sund. ku.dk, mariinaacrespo@gmail.com, cwang@sund.ku.dk, mitheis@ sund.ku.dk, susi@sund.ku.dk, and micali@sund.ku.dk. Samuel Yao Ahorhorlu, Centre for Tropical Clinical Pharmacology and Therapeutics, University of Ghana Medical School, University of Ghana, Ghana, E-mail: syahorhorlu@ug.edu.gh. Magatte Ndiaye, Service de Parasitologie-Mycologie, Faculté de Médecine, Université Cheikh Anta DIOP, Dakar, Senegal, E-mail: magou22000@yahoo.fr. John Lusingu, National Institute for Medical Research, Tanga Centre, Tanzania, E-mail: jpalusingu@gmail.com. Manoj Kumar Das, Field Unit, National Institute of Malaria Research, Ranchi, Jharkhand, India, E-mail: manojdas2@gmail.com. Subhash Singh, Indian Institute of Integrative Medicine, Jammu, India, E-mail: subhash0974@gmail.com. Susana Campino and Cally Roper, Faculty of Infectious and Tropical Diseases, London School of Hygiene and Tropical Medicine, London, United Kingdom, E-mail: susana.campino@Ishtm.ac.uk and cally.roper@Ishtm.ac.uk. Ole Lund, Genomic Epidemiology, Department of Bio and Health Informatics, Technical University of Denmark, Copenhagen, Denmark, E-mail: lund@bioinformatics.dtu.dk.

\section{REFERENCES}

1. WHO, 2020. World Malaria Report, 20 Years of Global Progress and Challenges. Geneva, Switzerland: World Health Organization. Available at: https://reliefweb.int/report/world/worldmalaria-report-2020. Accessed February 6, 2021.

2. Trape JF, 2001. The public health impact of chloroquine resistance in Africa. Am J Trop Med Hyg 64: 12-17.

3. Payne D, 1987. Spread of chloroquine resistance in Plasmodium falciparum. Parasitol Today 3: 241-246.

4. Trape JF, Pison G, Preziosi MP, Enel C, Desgrées du Loû A, Delaunay V, Samb B, Lagarde E, Molez JF, Simondon F, 1998. Impact of chloroquine resistance on malaria mortality. C R Acad Sci III 321: 689-697.

5. Kitua AY, 1999. Antimalarial drug policy: Making systematic change. Lancet 354 (Suppl): SIV32.

6. Gatton ML, Martin LB, Cheng Q, 2004. Evolution of resistance to sulfadoxine-pyrimethamine in Plasmodium falciparum. Antimicrob Agents Chemother 48: 2116-2123.

7. Roper C, Pearce R, Nair S, Sharp B, Nosten F, Anderson T, 2004. Intercontinental spread of pyrimethamine-resistant malaria. Science 305: 1124

8. Wootton JC, Feng X, Ferdig MT, Cooper RA, Mu J, Baruch DI, Magill AJ, Su X-Z, 2002. Genetic diversity and chloroquine selective sweeps in Plasmodium falciparum. Nature 418: 320323.

9. WHO, 2001. Antimalarial Drug Combination Therapy - Report of a WHO Technical Consultation. Geneva, Switzerland: World Health Organization. Available at: https://apps.who.int/iris/ bitstream/handle/10665/66952/WHO_CDS_RBM_2001.35. pdf?sequence=1. Accessed June 5, 2020.

10. WHO, 2019. World Malaria Report. Geneva, Switzerland: World Health Organization. Available at: https://www.who.int/ publications-detail/world-malaria-report-2019. Accessed January 29, 2021.

11. Huang F, Yan H, Xue JB, Cui YW, Zhou SS, Xia ZG, Abeyasinghe $R$, Ringwald $P$, Zhou XN, 2021. Molecular surveillance of pfert, pfmdr1 and pfk13-propeller mutations in Plasmodium falciparum isolates imported from Africa to China. Malar J 0: 73.

12. Lu $\mathrm{F}$ et al., 2017. Emergence of indigenous artemisinin-resistant Plasmodium falciparum in Africa. N Engl J Med 376: 991-993.

13. Bergmann $C$ et al., 2021. Increase in kelch 13 polymorphisms in Plasmodium falciparum, southern Rwanda. Emerg Infect Dis 27: 294-296.

14. Bwire GM, Ngasala B, Mikomangwa WP, Kilonzi M, Kamuhabwa AAR, 2020. Detection of mutations associated with artemisinin resistance at k13-propeller gene and a near complete return of chloroquine susceptible falciparum malaria in southeast of Tanzania. Sci Rep 10: 3500.

15. Tacoli C, Gai PP, Bayingana C, Sifft K, Geus D, Ndoli J, Sendegeya A, Gahutu JB, Mockenhaupt FP, 2016. Artemisinin resistance-associated k13 polymorphisms of Plasmodium falciparum in southern Rwanda, 2010-2015. Am J Trop Med Hyg 95: 1090-1093.

16. Uwimana A et al., 2020. Emergence and clonal expansion of in vitro artemisinin-resistant Plasmodium falciparum kelch13 r561h mutant parasites in Rwanda. Nat Med 26: 1602-1608.

17. Uwimana A et al., 2021. Association of Plasmodium falciparum kelch13 r561h genotypes with delayed parasite clearance in Rwanda: an open-label, single-arm, multicentre, therapeutic efficacy study. Lancet Infect Dis.

18. Asua $V$ et al., 2021. Changing prevalence of potential mediators of aminoquinoline, antifolate, and artemisinin resistance across Uganda. J Infect Dis 223: 985-994.

19. Ariey $\mathrm{F}$ et al., 2014. A molecular marker of artemisinin-resistant Plasmodium falciparum malaria. Nature 505: 50-55.

20. Zaw MT, Emran NA, Lin Z, 2018. Updates on k13 mutant alleles for artemisinin resistance in Plasmodium falciparum. J Microbiol Immunol Infect 51: 159-165.

21. Nag S, Dalgaard MD, Kofoed PE, Ursing J, Crespo M, Andersen LO, Aarestrup FM, Lund O, Alifrangis M, 2017. High throughput resistance profiling of Plasmodium falciparum infections based on custom dual indexing and Illumina next generation sequencing-technology. Sci Rep 7: 2398.

22. Ménard D et al., 2016. A worldwide map of Plasmodium falciparum k13-propeller polymorphisms. N Engl J Med 374: 24532464.

23. Taylor SM et al., 2015. Absence of putative artemisinin resistance mutations among Plasmodium falciparum in sub-Saharan Africa: a molecular epidemiologic study. J Infect Dis 211: 680-688.

24. Preston MD et al., 2014. A barcode of organellar genome polymorphisms identifies the geographic origin of Plasmodium falciparum strains. Nat Commun 5: 4052.

25. Das MK et al., 2017. Malaria epidemiology in an area of stable transmission in tribal population of Jharkhand, India. Malar $\mathrm{J}$ 16: 181.

26. Mkumbaye SI et al., 2017. The severity of Plasmodium falciparum infection is associated with transcript levels of var genes encoding endothelial protein c receptor-binding $P$. falciparum erythrocyte membrane protein 1. Infect Immun 85: e00841-16.

27. Ndiaye M, Sow D, Nag S, Sylla K, Tine RC, Ndiaye JL, Lo AC, Gaye O, Faye B, Alifrangis M, 2017. Country-wide surveillance of molecular markers of antimalarial drug resistance in Senegal by use of positive malaria rapid diagnostic tests. Am J Trop Med Hyg 97: 1593-1596.

28. WWARN, 2013. PCR and Sequencing for Genotyping of Candidate Plasmodium falciparum Artemisinin Resistance SNPs in the Kelch 13 Gene. Available at: https://www.wwarn.org/ tools-resources/procedures/pcr-and-sequencing-genotypingcandidate-plasmodium-falciparum-artemisinin. Accessed May 11, 2021.

29. Tyagi S, Das A, 2015. Mitochondrial population genomic analyses reveal population structure and demography of Indian Plasmodium falciparum. Mitochondrion 24: 9-21.

30. Tyagi S, Pande V, Das A, 2014. New insights into the evolutionary history of Plasmodium falciparum from mitochondrial genome sequence analyses of Indian isolates. Mol Ecol 23: 2975-2987.

31. Tyagi S, Pande V, Das A, 2014. Mitochondrial genome sequence diversity of Indian Plasmodium falciparum isolates. Mem Inst Oswaldo Cruz 109: 494-498.

32. Schoone GJ, Oskam L, Kroon NC, Schallig HD, Omar SA, 2000. Detection and quantification of Plasmodium falciparum in blood samples using quantitative nucleic acid sequence-based amplification. J Clin Microbiol 38: 4072-4075.

33. Conway DJ et al., 2000. Origin of Plasmodium falciparum malaria is traced by mitochondrial DNA. Mol Biochem Parasitol 111: 163-171.

34. Hughes AL, Verra F, 2001. Very large long-term effective population size in the virulent human malaria parasite Plasmodium falciparum. Proc Biol Sci 268: 1855-1860.

35. Tanabe $\mathrm{K}$ et al., 2010. Plasmodium falciparum accompanied the human expansion out of Africa. Curr Biol 20: 1283-1289.

36. Escalante AA, Barrio E, Ayala FJ, 1995. Evolutionary origin of human and primate malarias: evidence from the circumsporozoite protein gene. Mol Biol Evol 12: 616-626. 
37. Joy DA et al., 2003. Early origin and recent expansion of Plasmodium falciparum. Science 300: 318-321.

38. Talundzic E, Chenet SM, Goldman IF, Patel DS, Nelson JA, Plucinski MM, Barnwell JW, Udhayakumar V, 2015. Genetic analysis and species specific amplification of the artemisinin resistanceassociated kelch propeller domain in $P$. falciparum and $P$. vivax. PLoS One 10: e0136099.

39. Ocholla $\mathrm{H}$ et al., 2014. Whole-genome scans provide evidence of adaptive evolution in Malawian Plasmodium falciparum isolates. $J$ Infect Dis 210: 1991-2000.

40. Amambua-Ngwa A et al., 2019. Major subpopulations of Plasmodium falciparum in sub-Saharan Africa. Science 365: 813-816.

41. Mishra N, Bharti RS, Mallick P, Singh OP, Srivastava B, Rana R, Phookan S, Gupta HP, Ringwald P, Valecha N, 2016. Emerging polymorphisms in falciparum kelch 13 gene in northeastern region of India. Malar J 15: 583.

42. Rana R, Ranjit M, Bal M, Khuntia HK, Pati S, Krishna S, Das A, 2020. Sequence analysis of the k13-propeller gene in artemisinin challenging Plasmodium falciparum isolates from malaria endemic areas of Odisha, India: a molecular surveillance study. BioMed Res Int 2020: 8475246.

43. Chhibber-Goel J, Sharma A, 2019. Profiles of kelch mutations in Plasmodium falciparum across south Asia and their implications for tracking drug resistance. Int J Parasitol Drug Resist 11: 4958.

44. Torrentino-Madamet M et al., 2014. Limited polymorphisms in k13 gene in Plasmodium falciparum isolates from Dakar, Senegal in 2012-2013. Malar J 13: 472.

45. Madamet $\mathrm{M}$ et al., 2017. Absence of association between polymorphisms in the k13 gene and the presence of Plasmodium falciparum parasites at day 3 after treatment with artemisinin derivatives in Senegal. Int J Antimicrob Agents 49: 754-756.

46. Conrad MD, Bigira V, Kapisi J, Muhindo M, Kamya MR, Havlir DV, Dorsey G, Rosenthal PJ, 2014. Polymorphisms in k13 and falcipain-2 associated with artemisinin resistance are not prevalent in Plasmodium falciparum isolated from Ugandan children. PLoS One 9: e105690.

47. Wang Z, Shrestha S, Li X, Miao J, Yuan L, Cabrera M, Grube C, Yang Z, Cui L, 2015. Prevalence of k13-propeller polymorphisms in Plasmodium falciparum from China-Myanmar border in 2007-2012. Malar J 14: 168

48. Takala-Harrison S et al., 2015. Independent emergence of artemisinin resistance mutations among Plasmodium falciparum in Southeast Asia. J Infect Dis 211: 670-679.

49. Doolan DL, Dobaño C, Baird JK, 2009. Acquired immunity to malaria. Clin Microbiol Rev 22: 13-36.

50. Takala-Harrison S, Laufer MK, 2015. Antimalarial drug resistance in Africa: key lessons for the future. Ann N Y Acad Sci 1342: 6267.

51. Mukherjee A et al., 2017. Artemisinin resistance without pfkelch13 mutations in Plasmodium falciparum isolates from Cambodia. Malar J 16: 195

52. Sutherland CJ et al., 2017. Pfk13-independent treatment failure in four imported cases of Plasmodium falciparum malaria treated with artemether-lumefantrine in the United Kingdom. Antimicrob Agents Chemother 61: e02382-e02316.

53. Adams T, Ennuson NAA, Quashie NB, Futagbi G, Matrevi S, Hagan OCK, Abuaku B, Koram KA, Duah NO, 2018. Prevalence of Plasmodium falciparum delayed clearance associated polymorphisms in adaptor protein complex 2 mu subunit (pfap2mu) and ubiquitin specific protease 1 (pfubp1) genes in Ghanaian isolates. Parasit Vectors 11: 175

54. Ofori MF, Kploanyi EE, Dickson EK, Kyei-Baafour E, Mensah BA, Koram KA, Abuaku BK, Gyabaa S, Tetteh M, Ghansah A, 2021. Ex vivo sensitivity profile of Plasmodium falciparum clinical isolates to a panel of antimalarial drugs in Ghana 13 years after national policy change. Infect Drug Resist 14: 267-276. 